\title{
openheart Rapid use of high-sensitive cardiac troponin I for ruling-in and ruling-out of acute myocardial infarction (RACING-MI): study protocol
}

\author{
Camilla Bang, ${ }^{1,2}$ Camilla Hansen, ${ }^{1,2}$ Kasper Glerup Lauridsen, ${ }^{1,2}$ \\ Christian Alcaraz Frederiksen, ${ }^{3}$ Morten Schmidt, ${ }^{4,5}$ Tage Jensen, ${ }^{2}$ Nete Hornung, ${ }^{6}$ \\ Bo Løfgren ${ }^{\oplus 1,2}$
}

\begin{abstract}
- Additional material is published online only. To view please visit the journal online (http://dx.doi.org/10.1136/ openhrt-2018-000995).
\end{abstract}

To cite: Bang C, Hansen C, Lauridsen KG, et al. Rapid use of high-sensitive cardiac troponin I for ruling-in and ruling-out of acute myocardial infarction (RACING-MI): study protocol. Open Heart 2019;6:e000995. doi:10.1136/

openhrt-2018-000995

$\mathrm{CB}$ and $\mathrm{CH}$ contributed equally.

$\mathrm{CB}$ and $\mathrm{CH}$ are joint first authors.

Received 23 December 2018 Revised 18 February 2019 Accepted 4 March 2019

Check for updates

(c) Author(s) (or their employer(s)) 2019. Re-use permitted under CC BY-NC. No commercial re-use. See rights and permissions. Published by BMJ.

For numbered affiliations see end of article.

Correspondence to Professor Bo Løfgren; bl@clin. au.dk

\section{ABSTRACT}

Introduction The European Society of Cardiology has suggested an accelerated algorithm for ruling-in and ruling-out myocardial infarction (MI) with high-sensitive cardiac troponin (hs-cTn) measured at admission (0 hour) and after 1 hour ( 1 hour) as an alternative to standard measurements at 0 hour and 3 hours. However, the 0 hour 1 hour algorithm has only been tested in a limited amount of patient cohorts and not for all hs-cTn assays. Moreover, it is unknown if Ml can be ruled-out faster than 1 hour. In this single-centre, clinical trial, we will investigate whether Ml safely can be ruled-in or ruled-out after 30 min and 1 hour.

Methods and analysis Patients with chest pain suggestive of Ml admitted to the emergency department will be subjected to hs-cTn measurements at the following time points: 0 hour, $30 \mathrm{~min}, 1$ hour and 3 hours. Chest pain characteristics will be recorded. In total, 1000 patients with all four blood samples will be included. The diagnostic algorithms will be derived based on the first 500 patients and validated in the subsequent 500 patients. The primary endpoint is the negative predictive value of the 0 hour $/ 30 \mathrm{~min}$ and the 0 hour $/ 1$ hour algorithms. Secondary endpoints include positive predictive value, sensitivity and specificity. Results will be compared with the standard 0 hour/3 hour algorithm.

Ethics and dissemination Oral and written informed consent will be obtained from all patients. The trial is approved by The Regional Committee on Health Research Ethics and the Danish Data Protection Agency. Data will be disseminated and submitted to peer-reviewed scientific journals and meetings irrespective of study outcome. Trial registration number NCT03634384

\section{INTRODUCTION}

Chest pain is a key symptom of myocardial infarction (MI) and accounts for 5\%-20\% of all non-surgery emergency department (ED) contacts. ${ }^{1-3}$ Nonetheless, only 1 in 10 patients admitted to the ED with chest pain are diagnosed with MI. ${ }^{1-3}$ Rapid 'rule-in' and 'rule-out' of MI are essential to minimise unnecessary bed allocation and time to

\section{Key questions}

What is already known about this subject?

- Recently, European Society of Cardiology introduced an assay-specific 0 hour/1 hour algorithm measuring troponin at admission and 1 hour later for accelerated rule-in and rule-out of acute myocardial infarction.

- Several countries have not yet implemented the algorithm because it has only been validated in few patient cohorts and for certain high-sensitive cardiac troponin assays. Moreover, it is unknown if myocardial infarction can be ruled out earlier than at 1 hour.

What does this study add?

- This trial will provide data on the performance of the 0 hour/1 hour algorithm in a patient cohort that differs from already existing studies.

- To reflect clinical practice, this trial will use ad hoc in addition to batch analysis of blood samples. Furthermore, this is the first large-scale trial to investigate measurements of high-sensitive cardiac troponin already 30 min after admission blood samples. This will allow investigation of a novel 0 hour/30 min algorithm, which has never been done before.

How might this impact on clinical practice?

- This trial will contribute with important knowledge on the performance of accelerated rule-in and ruleout algorithms and offers insights into early troponin kinetics. If the results of this trial support the use of the 0 hour 11 hour algorithm, this will add to the overall external validity of accelerated rule-in and rule-out algorithms and potentially favour a wide clinical implementation. In addition, we will be able to evaluate the performance of a novel 0 hour $/ 30$ min algorithm allowing for even faster rule-out of acute myocardial infarction.

discharge for patients without MI and ensure prompt treatment for patients with MI. ${ }^{4}$

In the absence of ST-segment elevation myocardial infarction (STEMI) on the 
ECG, the European Society of Cardiology (ESC) recommend repeated measurements of high-sensitive cardiac troponin (hs-cTn) at presentation (0 hour) and 3 hours after presentation to rule-in or -out MI (the 0 hour $/ 3$ hour algorithm). ${ }^{56}$ An accelerated algorithm after 1 hour (1 hour) has been suggested, ${ }^{7-14}$ but remains largely to be implemented in clinical practice as it has mainly been investigated in patient cohorts originating from the APACE trial. ${ }^{15}$ Moreover, data on patients undergoing transcoronary septal ablation of septal hypertrophy have demonstrated an increase in troponin concentrations measured by a hs-cTn assay only $15 \mathrm{~min}$ after induction of myocardial injury. ${ }^{16}$ This finding indicates that it may be possible to evaluate troponin dynamics earlier than the 0 hour/1 hour algorithm.

We will investigate if high-sensitive cardiac troponin I (hs-cTnI) measurements at $30 \mathrm{~min}$ or 1 hour after initial hs-cTn measurements can safely rule-in or rule-out MI.

\section{METHODS}

\section{Study design}

We will conduct a single-centre, clinical trial of patients with chest pain suggestive of MI admitted to the ED. There will be no randomisation. Patients will be treated according to standard Danish practice, which follows the guidelines from ESC. ${ }^{6}$ To avoid any ethical concerns on whether or not to act on hs-cTnI results from the $30 \mathrm{~min}$ or 1 hour blood samples, the treating physician will be blinded to the 30 min and 1 hour results until the 3 hour results become available. As a consequence, the treating physician can act only on hs-cTnI at 0 hour and 3 hours, according to current clinical practice.

\section{Study setting}

In Denmark, patients are examined in the prehospital setting for symptoms and clinical findings suggestive of MI and subsequently admitted to a public hospital. ${ }^{17}$ Patients with STEMI or elevated point-of-care cardiac troponin measured in the ambulance will be admitted directly to an invasive heart centre. In the absence of STEMI or elevated point-of-care cardiac troponin, patients are referred to a regional hospital for observation and diagnostic workup. Currently, patients admitted to Danish hospitals under suspicion of MI are evaluated in accordance with the 0 hour $/ 3$ hour troponin algorithm.

RACING-MI will be carried out in the ED at Randers Regional Hospital, Denmark. As a consequence, the expected incidence of MI in our population will be relatively low due to the prehospital referral of patients with positive point-of-care measurements to the nearest university hospital. Inclusion was started on 21 November 2016 and will continue until fulfilment of the sample size. The trial is registered at http://www.clinicaltrials.gov (trial registration number: NCT03634384).

\section{Eligibility and exclusion criteria}

All patients with chest pain suggestive of MI will be eligible for inclusion. Exclusion criteria are defined as STEMI at
Table 1 Trial components and timeline for data collection of the RACING-MI study

\begin{tabular}{|c|c|c|c|c|c|}
\hline $\begin{array}{l}\text { Trial } \\
\text { components }\end{array}$ & $\begin{array}{l}\text { At } \\
\text { admission } \\
\text { (0 hour) }\end{array}$ & $\begin{array}{l}\text { At } 30 \\
\mathrm{~min}\end{array}$ & $\begin{array}{l}\text { At } 1 \\
\text { hour }\end{array}$ & $\begin{array}{l}\text { At } 3 \\
\text { hours }\end{array}$ & $\begin{array}{l}30 \text { days } \\
\text { after } \\
\text { discharge }\end{array}$ \\
\hline Patient screening & $x$ & & & & \\
\hline Informed consent & $x$ & & & & \\
\hline ECG & $x$ & & & & \\
\hline Vital parameters & $x$ & & & & \\
\hline Trial blood samples & $x$ & $x$ & $x$ & $x$ & \\
\hline $\begin{array}{l}\text { Routine blood } \\
\text { samples }\end{array}$ & $x$ & & & $x$ & \\
\hline Questionnaire & $x$ & & & & \\
\hline Medication* & $x$ & & & & \\
\hline Follow-up & & & & & $X$ \\
\hline
\end{tabular}

${ }^{*}$ Registered as medication use at the time of admission.

admission, age $<18$ years, pregnancy and kidney failure requiring dialysis. As part of standard procedure, the admitting physician evaluates all patients, including ECG, on admission and decides whether to continue observation for MI or not. The admitting healthcare staff will hereafter assess the patient for trial eligibility.

\section{Admission data}

Time of first admission contact as registered in the electronic health record will be registered for each patient. The time of admission blood samples defines 0 hour. Table 1 presents an overview of trial components and data collection time points. All patients will receive routine clinical cardiac assessment at admission (ECG and vital parameters such as blood pressure, pulse oximetry, respiratory frequency and temperature).

\section{Blood tests}

The admitting nurse will request the trial blood samples. Each patient will undergo serial blood sampling at 0 hour, 30 min, 1 hour and 3 hours (table 1 ). The admission hs-cTnI sample ( 0 hour) will be included in the standardised admission blood samples. Blood samples will be obtained by standard venous blood sampling. If a suitable peripheral venous catheter is available and venipuncture is unsuccessful or considered harmful by the patient, the venous catheter may be used for blood sampling. In such cases, the first $10 \mathrm{~mL}$ blood obtained will be discarded at the 30 min and 1 hour blood draw to avoid haemolysis in the blood samples.

A timer will be set by the medical laboratory technician at the 0 hour blood sampling to notify the admitting physician or a research assistant of when to obtain the 30 min and 1 hour blood samples. The exact time of each blood draw will be registered on a data sheet and on the blood requisition forms. The $30 \mathrm{~min}$ and 1 hour trial blood samples will be placed in a container and collected by a medical laboratory technician at the 3 hour blood sampling. Hs-cTnI will be measured in the central 
laboratory for each blood sampling (0 hour, $30 \mathrm{~min}, 1$ hour and 3 hours). An additional $10 \mathrm{~mL}$ lithium heparin and $4 \mathrm{~mL}$ serum for all four time-points will be stored in a research biobank.

\section{Questionnaire}

Patients will be asked to complete a 19-item questionnaire on their chest pain characteristics and cardiovascular risk profile (online supplementary table 1). The questionnaire covers (1) time of chest pain onset and peak, (2) chest pain characteristics (localisation, radiation, sensation), (3) additional symptoms at presentation (nausea, abdominal pain, syncope, dyspnoea and palpitations), (4) life style factors such as physical activity, smoking status, height and weight, (5) family history of MI and (6) whether the patient believes that his or her symptoms are caused by MI (yes/no). If no, the patient will be asked to elaborate on expected final diagnosis.

\section{Data entry}

A research assistant will record baseline characteristics for all patients from the electronic patient record. Data will include time of admission, age, sex, vital parameters on admission, admission blood test results, the four hs-cTnI results, ECG description by the physician, medication use on admission, previous MI and comorbidity (online supplementary table 2). The investigator will stratify patients according to HEART risk score ${ }^{18}$ when all data on admission are available, including initial hs-cTnI values, and record the preliminary final diagnosis.

\section{Adjudication}

After patient inclusion, two physicians will independently adjudicate the final diagnosis for each patient. In case of discrepancy, a third physician will be consulted, and the final diagnosis will be determined by consensus. All available clinical information (eg, patient history, physical examination, laboratory testing, radiology imaging, ECG, echocardiography and results from coronary angiography) pertaining to the patient from admission at the ED to 30 days after discharge, will be used as part of the adjudication. Follow-up will include major adverse cardiac events defined in accordance with previous studies on the HEART score, that is, MI, percutaneous coronary intervention, coronary artery bypass grafting and all-cause mortality. ${ }^{18-20}$ Non-fatal outcomes will be identified from hospital discharge diagnoses. ${ }^{17}$ Mortality will be identified from the Danish Civil Registration System. ${ }^{21}$ Patients will be classified with a final diagnosis of MI based on the fourth universal definition of MI. ${ }^{22}$ The final diagnosis of MI will be based on a rise and/or fall of troponin values with at least one value above the 99th percentile combined with evidence of myocardial ischaemia. In cases with elevated troponin with no troponin dynamics or no signs of myocardial ischaemia, the case will be discussed and a consensus decision will be made in accordance with guidelines. ${ }^{22}$

\section{Clinical biochemistry}

All trial blood samples will be centrifuged at $1500 \mathrm{~g}$ for 10 min. Hs-cTnI is measured instantly from the $4 \mathrm{~mL}$ lithium heparin tube. Plasma from the $10 \mathrm{~mL}$ heparin and $4 \mathrm{~mL}$ serum tubes will be kept frozen at $-80^{\circ} \mathrm{C}$ for later analysis.

Troponin analyses will be performed in accordance with routine laboratory methodology on ADVIA Centaur using the high-sensitivity troponin I (TNIH) assay (Siemens Healthcare GmbH, Erlangen, Germany). The method is accredited according to the DS/EN ISO 15189:2013 accreditation standard. The limit of detection for the TNIH assay is $2.21 \mathrm{ng} / \mathrm{L}$, the 99 th percentile cut-off point is $47.34 \mathrm{ng} / \mathrm{L}$ and the coefficient of variation is less than $10 \%$ at $4.46 \mathrm{ng} / \mathrm{L}$.

\section{Endpoints}

Data from the first 500 patients will be used to derive diagnostic 0 hour $/ 30$ min and 0 hour/ 1 hour algorithms, which subsequently will be validated in the last 500 patients. For the 0 hour $/ 1$ hour algorithm, data from the total cohort will also be used to test and further validate already existing 0 hour $/ 1$ hour algorithm cut-off values. ${ }^{23}$

The primary endpoint will be the negative predictive value of the 0 hour $/ 30 \mathrm{~min}$ and the 0 hour/ 1 hour algorithms. Secondary endpoints will include positive predictive value and sensitivity and specificity at $30 \mathrm{~min}$ and 1 hour. Results will be compared with the standard 0 hour/3 hour algorithm.

\section{Derivation of the algorithms}

The 1 hour algorithm for rapid rule-in and rule-out of acute MI has previously been described. ${ }^{70112425} \mathrm{~A}$ $30 \mathrm{~min}$ algorithm has not previously been described. In brief, we will derive the two algorithms by incorporating both baseline and absolute changes in hs-cTn levels within the first $30 \mathrm{~min}$ ( 0 hour $/ 30 \mathrm{~min}$ algorithm) and 1 hour ( 0 hour/ 1 hour algorithm). Rule-out will be defined as hs-cTn level changes within the first $30 \mathrm{~min}$ or 1 hour less than the trial derived hs-cTn cut-off values and rule-in as hs-cTn changes greater than the trial derived hs-cTn cut-off values. Patients fulfilling neither of the above criteria for rule-in or rule-out will be stratified to an 'observational zone' requiring further diagnostic work-up.

\section{Sample size}

The trial is designed to enrol 1000 patients with complete blood samples at 0 hour, $30 \mathrm{~min}, 1$ hour and 3 hours. Pilot study calculations estimate an expected cumulative incidence of MI in our trial cohort of only 7\%. Assuming a negative predictive value of $99.7 \%$ in the rule-out group, a distribution with $7 \%$ patients in the rule-in group and $20 \%$ assigned to the observational zone (patients not stratified to either the rule-out or rule-in group), enrolment of 500 patients (with complete data) in both the derivation 
and validation cohorts will provide an acceptable lower boundary of $98.2 \%$ of the two-sided $95 \%$ CI. ${ }^{81025}$

\section{Statistical analysis}

Data will be analysed for normality using QQ-plots and histograms. Normally distributed continuous variables will be presented as mean $\pm \mathrm{SD}$ and non-normal as median (quartile 1; quartile 3). Categorical data will be presented as numbers and proportions. Statistical differences will be measured using appropriate parametric and non-parametric tests.

All hypothesis testing will be 2-tailed with a $\mathrm{p}<0.05$ considered to be statistically significant. Statistical analysis will be performed using R statistical software, V.3.4.0.

\section{Strengths}

This will be the first large-scale trial to measure hs-cTn already 30 min after admission blood sampling and the first trial to introduce a 0 hour/30 min diagnostic algorithm. Only one small trial including 71 patients with high risk of MI has investigated $30 \mathrm{~min}$ blood sampling using a single cut-off with no standard 3 hour hs-cTn reference. ${ }^{26}$

In addition, this trial will provide data on the performance of the 0 hour/1 hour algorithm from a patient cohort that differs from already existing studies. ${ }^{15}$ As a result, this may add to the external validity of faster rule-in/ rule-out algorithms of MI. This trial will use ad hoc in addition to batch analysis of blood samples. It will then be possible to investigate the difference between the analytical precisions of ad hoc and batch analysis. ${ }^{27}$ In addition, a research biobank will be established with additional blood samples for each patient at all time points. The biobank will provide the opportunity to validate different troponin assays and further investigate the patient cohort.

\section{Limitations}

Potential limitations of the present trial should be considered. Currently, it is unknown if repeated blood sampling after $30 \mathrm{~min}$ is feasible in clinical practice. However, possibilities exist, for example, (1) implementation of automatic electronic notification as for the standard 3 hour blood sample and (2) prehospital blood draw in the ambulance, which is handed over for analysis on arrival.

This trial is not a randomised controlled trial. However, the patients will be used as their own control group and will not affect the measures of endpoints. In addition, it allows us to safely investigate the novel 0 hour/30 min algorithm.

\section{Ethics and dissemination}

Oral and written informed consent will be obtained from all patients in accordance with the Danish Health Act and the Declaration of Helsinki. The trial is approved by The Regional Committee on Health Research Ethics (no. 1-1072-213-16) and the Danish Data Protection Agency (no. 1-16-02-530-16). The admitting healthcare staff will obtain the informed consent prior to the blood sampling.

The findings of this trial will be disseminated by peer reviewed publications, presentation at national and international meetings as well as in other relevant channels.

\section{Author affiliations}

${ }^{1}$ Research Center for Emergency Medicine, Aarhus University Hospital, Aarhus, Denmark

2Department of Internal Medicine, Randers Regional Hospital, Randers, Denmark ${ }^{3}$ Department of Cardiology, Aarhus University Hospital, Aarhus, Denmark ${ }^{4}$ Department of Cardiology, Regional Hospital West Jutland, Herning, Denmark ${ }^{5}$ Department of Clinical Epidemiology, Aarhus University Hospital, Aarhus, Denmark ${ }^{6}$ Department of Biochemistry, Regional Hospital West Jutland, Herning, Denmark

Acknowledgements The authors thank all the patients for their willingness to participate in the trial. We also thankfully acknowledge the dedicated work of all healthcare staff at Randers Regional Hospital involved in the trial. We specifically wish to thank Maja Morsing, Birgit Winther Friis, Josephine Johnsen, Alexandra Uglebjerg Pedersen and Martin Bjergskov Thomsen for their assistance with biobank establishment and data collection.

Contributors $\mathrm{CH}, \mathrm{CB}, \mathrm{KGL}, \mathrm{CAF}, \mathrm{MS}, \mathrm{NH}$ and $\mathrm{BL}$ : conception and design. $\mathrm{CH}$ and $\mathrm{CB}$ : funding acquisition. $\mathrm{CB}, \mathrm{CH}, \mathrm{NH}, \mathrm{TJ}, \mathrm{BL}$ : acquisition of data. $\mathrm{CB}, \mathrm{CH}, \mathrm{KGL}, \mathrm{MS}$, $\mathrm{CAF}, \mathrm{BL}$ : analysis of data. $\mathrm{CH}, \mathrm{CB}, \mathrm{KGL}, \mathrm{CAF}, \mathrm{MS}, \mathrm{NH}$ and $\mathrm{BL}$ : interpretation of data $\mathrm{CB}$ and $\mathrm{CH}$ : drafting of manuscript. $\mathrm{CH}, \mathrm{CB}, \mathrm{KGL}, \mathrm{CAF}, \mathrm{MS}, \mathrm{TJ}, \mathrm{NH}$ and $\mathrm{BL}$ : revising manuscript of critically important intellectual content. $\mathrm{CH}, \mathrm{CB}, \mathrm{KGL}, \mathrm{CAF}, \mathrm{MS}, \mathrm{TJ}, \mathrm{NH}$ and $\mathrm{BL}$ : final approval of manuscript. $\mathrm{CB}$ and $\mathrm{CH}$ contributed equally to this trial. All authors had full access to the data and agree to take responsibility for all aspects of the work.

Funding This work was supported by the Department of Internal Medicine, the Department of Biochemistry and the Emergency Department, Randers Regional Hospital, together with the Research Center for Emergency Medicine, Aarhus University Hospital. Moreover, funding has been provided by the Grosserer L. F. Foghts Foundation, Boserup Foundation, Helga and Peter Korning Foundation, Holger and Ruth Hesse's Memorial Foundation, Henry and Astrid Møller Foundation, the Højmosegård Grant, 'Spiremidler' (start-up grant), Oda and Hans Svenningsen's Foundation, The Foundation of 1870 and AP Møller Foundation. Grant supports are provided by Randers Regional Hospital and Aarhus University. TNIH assays were provided by Siemens Healthcare A/S, Ballerup, Denmark.

Competing interests None declared.

Patient consent for publication Not required.

Provenance and peer review Not commissioned; externally peer reviewed.

Data sharing statement For permission to use data from the RACING-MI study contact principal investigator, Professor Bo Løfgren, MD, PhD, FESC, FAHA on mail bl@clin.au.dk.

Open access This is an open access article distributed in accordance with the Creative Commons Attribution Non Commercial (CC BY-NC 4.0) license, which permits others to distribute, remix, adapt, build upon this work non-commercially, and license their derivative works on different terms, provided the original work is properly cited, appropriate credit is given, any changes made indicated, and the use is non-commercial. See: http://creativecommons.org/licenses/by-nc/4.0/.

\section{REFERENCES}

1. Bhuiya F, Pitts S, McCaig L. Emergency department visits for chest pain and abdominal pain- United States, 1999-2008. NCHS data brief 2010;43:1-8.

2. Barfod C, Lauritzen M, Danker J, et al. The formation and design of the 'Acute Admission Database'- a database including a prospective, observational cohort of 6279 patients triaged in the emergency department in a larger Danish hospital. Scand J Trauma Resusc Emerg Med 2012;20.

3. Foy AJ, Filippone L. Chest Pain Evaluation in the emergency department. Med Clin North Am 2015;99:835-47.

4. Forberg JL, Henriksen LS, Edenbrandt L, et al. Direct hospital costs of chest pain patients attending the emergency department: a retrospective study. BMC Emerg Med 2006;6.

5. Apple FS, Jaffe AS, Collinson P, et al. IFCC educational materials on selected analytical and clinical applications of high sensitivity cardiac troponin assays. Clin Biochem 2015;48:201-3.

6. Roffi M, Patrono C, Collet J-P, et al. 2015 ESC guidelines for the management of acute coronary syndromes in patients presenting without persistent ST-segment elevation: Task Force for the 
management of acute coronary syndromes in patients presenting without persistent ST-segment elevation of the European Society of cardiology (ESC). Eur Heart $J$ 2016;37:267-315.

7. Reichlin T, Schindler C, Drexler B, et al. One-hour rule-out and rule-in of acute myocardial infarction using high-sensitivity cardiac troponin T. Arch Intern Med 2012;172:1211-8.

8. Jaeger C, Wildi K, Twerenbold R, et al. One-hour rule-in and rule-out of acute myocardial infarction using high-sensitivity cardiac troponin I. Am Heart J 2016;171:92-102.

9. Jaffe AS, White H, Injury R-IM. Ruling-In myocardial injury and Ruling-Out myocardial infarction with the European Society of cardiology 1-hour algorithm. Circulation 2016;134:1542-5.

10. Mueller C, Giannitsis E, Christ M, et al. Multicenter evaluation of a 0-Hour/1-Hour algorithm in the diagnosis of myocardial infarction with high-sensitivity cardiac troponin T. Ann Emerg Med 2016;68:76-87.

11. Neumann JT, Sörensen NA, Schwemer T, et al. Diagnosis of myocardial infarction using a high-sensitivity troponin I 1-hour algorithm. JAMA Cardiol 2016;1:397-404.

12. Pickering JW, Greenslade JH, Cullen L, et al. Assessment of the European Society of cardiology 0-Hour/1-Hour algorithm to Rule-Out and Rule-In acute myocardial infarction. Circulation 2016;134:1532-41.

13. Boeddinghaus J, Nestelberger T, Twerenbold R, et al. Direct comparison of 4 very early Rule-Out strategies for acute myocardial infarction using high-sensitivity cardiac troponin I. Circulation 2017;135:1597-611.

14. Neumann JT, Sörensen NA, Ojeda F, et al. Early diagnosis of acute myocardial infarction using high-sensitivity troponin I. PLoS One 2017;12:e0174288.

15. ClinicalTrials.gov. Identifier NCT00470587. Mueller (MD): National Library of Medicine - Advantageous Predictors of Acute Coronary Syndromes Evaluation (APACE) Study (APACE), 2007. Available: https://clinicaltrials.gov/ct2/show/NCT00470587?term= NCT00470587\&rank=1 [Accessed 10 Feb 2017].

16. Liebetrau $\mathrm{C}$, Möllmann $\mathrm{H}$, Nef $\mathrm{H}$, et al. Release kinetics of cardiac biomarkers in patients undergoing transcoronary ablation of septal hypertrophy. Clin Chem 2012;58:1049-54.
17. Schmidt M, Schmidt SAJ, Sandegaard JL, et al. The Danish national patient Registry: a review of content, data quality, and research potential. Clin Epidemiol 2015;7:449-90.

18. Six AJ, Backus BE, Kelder JC. Chest pain in the emergency room: value of the heart score. NHJL 2008;16:191-6.

19. Byrne C, Toarta C, Backus B, et al. The heart score in predicting major adverse cardiac events in patients presenting to the emergency department with possible acute coronary syndrome: protocol for a systematic review and meta-analysis. Systematic Reviews

2018;7.

20. Backus BE, Six AJ, Kelder JC, et al. Chest pain in the emergency room. Critical Pathways in Cardiology: A Journal of Evidence-Based Medicine 2010;9:164-9

21. Schmidt M, Pedersen L, Sørensen HT. The Danish civil registration system as a tool in epidemiology. Eur J Epidemiol 2014;29:541-9.

22. Thygesen K, Alpert JS, Jaffe AS, et al. Fourth universal definition of myocardial infarction (2018). Eur Heart J 2019;40:237-69.

23. Boeddinghaus J, Twerenbold R, Nestelberger T, et al. Clinical validation of a novel high-sensitivity cardiac troponin I assay for early diagnosis of acute myocardial infarction. Clin Chem 2018;64:1347-60.

24. Rubini Gimenez M, Twerenbold R, Jaeger C, et al. One-hour rule-in and rule-out of acute myocardial infarction using high-sensitivity cardiac troponin I. Am J Med 2015;128:861-70.

25. Reichlin T, Twerenbold R, Wildi K, et al. Prospective validation of a 1-hour algorithm to rule-out and rule-in acute myocardial infarction using a high-sensitivity cardiac troponin T assay. CMAJ 2015;187:E2 43-E252.

26. Yokoyama H, Higuma T, Endo T, et al. "30-minute-delta" of highsensitivity troponin I improves diagnostic performance in acute myocardial infarction. J Cardiol 2018;71:144-8.

27. Lauridsen KG, Revsholm J, Løfgren B. Appropriate use of highsensitivity cardiac troponin levels in patients with suspected acute myocardial infarction. JAMA Cardiol 2017;2:228-9. 